\title{
Supplementary information in schematic concept formation
}

\author{
JEAN F. TRACY, UNIVERSITY OF TEXAS AT ARLINGTON \\ SELBY H. EVANS, TEXAS CHRISTIAN UNIVERSITY
}

Five conditions of supplementary information, including exemplars and knowledge of results, were used in a four category sorting task requiring acquisition of a schematic concept. While most Ss showed some amount of concept acquisition, different amounts of supplementary information did not differentially affect their performance. The results were consistent with the construct of schematic concept formation.

A basic supposition of schema theory is that schema learning can occur on the basis of the information provided in the stimuli, without the aid of external or supplementary information about the schema (Evans, 1967a). In the context of schema theory, a schema is a set of rules which would serve as instructions for producing (in essential aspects) a population prototype. A schema family is a population of objects, all of which can be efficiently described by the same schema rules. Evans defined schematic concept formation as the "development of the ability to assign objects to their corresponding schema families on the basis of the information derived from perceiving the objects, without any other source of information as to the appropriate categorization, and without prior familiarization with the relevant schema." (1967a, p. 88). The purpose of the present study was to investigate the effects on schematic concept formation of information supplementary (SI) to that provided in the stimulus, information such as knowledge of results.

Although schematic concept formation seems to occur with or without SI (Evans, 1964; Evans \& Edmonds, 1966; Evans \& Arnoult, 1967; Rosser, 1967), there have been no systematic studies of the effects of different amounts of SI. Even though schematic concept formation can occur without SI, increasing amounts of SI might be expected to facilitate learning. This expectation receives support from a study by Edmonds, Mueller, \& Evans (1966), in which both SI and no SI conditions were used with a schematic oddity problem task. The performance of the SI group was not significantly different in the final block of trials from that of the no SI group, but the SI group improved more rapidly in the early stages of learning.

Although a free sorting task has been the principal method for studying schematic concept formation, this method requires a specialized scoring procedure (Evans \& Arnoult, 1967). Because this procedure leads to scores which are not comparable to those obtained under conditions of $\mathrm{SI}_{\mathrm{a}}$ the free sorting task was not used in this study. Five levels of SI were used; the minimum SI consisted of a single exemplar for each schema. Other levels of SI were produced by incomplete and complete knowledge of results, and by combinations of these conditions with the exemplar condition. The task consisted of sorting a deck of cards which contained a random ordering of instances from four different schema families. Each $S$ sorted the deck twice. Performance was measured both by the number of instances sorted correctly and by the time required to sort the entire deck.

The experimental hypotheses were: (1) Increasing amounts of SI should lead to better performance; (2) Performance should be better on the second sort than on the first; and (3) All groups should perform above chance levels.

Subjects

Fifty general psychology students at the University of Texas at Arlington served as Ss and all were randomly assigned to treatment groups and to order of presentation.

\section{Supplementary Information}

Five conditions of SI were used: (1) Exemplar SI, in which a correct example of each schema was present throughout the task; (2) Incomplete SI, in which $\mathbf{E}$ told $\mathrm{S}$ whether each response was correct or not (Patterns which were incorrectly placed were discarded, so that each stack had a correct example in view.); (3) Complete SI, in which $\mathrm{E}$ corrected each wrong response by telling $\mathrm{Ss}$ where the card should go (The $S$ then placed the pattern correctly.); (4) Exemplar + Incomplete SI, in which Conditions (1) and (2) were combined; and (5) Exemplar + Complete SI, in which Conditions (1) and (3) were combined. Each group which received exemplars was given the same exemplars so that no variance would be due to different exemplars.

Stimuli

Sixty VARGUS 7 patterns $^{2}$ (Evans, 1967b), 15 from each of four different schemata, were obtained at $70 \%$ redundancy. The patterns were secured individually to $5 \times 7$ cards and arranged in a random permutation. Two permutations were used; each was used with half the Ss in each group.

Procedure

Ss were individually given the sorting task while seated at a desk with $E$ in a quiet room. Ss were instructed to find $E^{\prime}$ 's rule so that they could sort the patterns correctly into four equal stacks. Instructions 
were given to each SI group that were specific to that experimental condition. Presentation of and placement of one stimulus card was counted as a trial. Results and Discussion

Separate one way analyses of variance were performed for the two dependent variables, number correct and time to sort, on the two sortings through the cards. No significant effects were found. Thus, Hypothesis (1) was not supported. As a test of Hypothesis (2), the first and second sortings for each group were compared with respect to either dependent variable by means of correlated $t$ tests. The results must be considered as qualified by the fact that they represent three significant outcomes from 10 comparisons. Improvement in number correct appeared under Exemplar SI $(t=2.87, d f=9, p<.02)$ and under Exemplar + Incomplete SI $(t=2.78, \mathrm{df}=9, \mathrm{p}<.05)$. Only the $E x-$ emplar + Incomplete SI group showed a significant difference $(t=2.59, d f=9, p<.02)$ in time to sort. In view of the small number of significant outcomes, Hypothesis (2) must be regarded as not supported.

Hypothesis (3) was tested by means of the binomial distribution. With a probability of .25 of being correct by chance, a total score of 21 correct out of 60 is significantly above chance at the .05 level of confidence. If the two runs by each $S$ are considered separately, there were 100 runs altogether; and of these, 74 were significantly above chance. Thus, Hypothesis (3) was supported.

The lack of significant differences due to SI suggests that SI is less important in schematic concept formation than in classical (didactic) concept formation. The results agree with the supposition of schema theory (Evans, 1967a) that schema learning can occur with or without supplementary information. The result is somewhat remarkable, however, in view of the important role that has traditionally been assigned to knowledge of results in interpretations of learning.

The failure of knowledge of results to improve performance may have resulted from particular characteristics of the VARGUS 7 patterns. The criterial attributes of these patterns are quite elusive, and informal questioning in this and similar studies has indicated that few Ss are able clearly to verbalize the basis on which they were sorting. Furthermore, since the attributes are probabilistic, no one of them is completely reliable. Observations have also sug- gested that many Ss find the task rather frustrating. Knowledge of results, in the presence of elusive and unreliable attributes, may have increased this frustration enough to nullify any beneficial effects. The failure to improve on the second sort may have a similar explanation, although fatigue is another possibility in this case. Certain aspects of the procedure, such as number of trials and amount of deviation of exemplars, might have contributed to the lack of significant differences between SI groups.

The fact that most Ss scored above chance shows that statistically defined concepts can be acquired in a four category sorting task. The successful performance in the Exemplar condition demonstrates again that knowledge of results is not needed for concept formation with schema defined concepts. The failure of knowledge of results to produce substantially better performance than was produced by exemplars alone raises the question of whether knowledge of results, even when it is given, contributes substantially to the acquisition of schema defined concepts. These conclusions give added support to the construct of schematic concept formation.

\section{References}

EDMONDS, E. M., MUELLER, M. R., \& EVANS, S. H. Effects of knowledge of results on mixed schema discrimination. Psychon. Sci, 1966, 6, 377-378

EVANS, S. H. A model for perceptual category formation. Unpublished doctoral dissertation, Texas Christian University, 1964.

EVANS, S. H. A brief statement of schema theory. Psychon. Sci, 1967a, 8, 87-88.

EVANS, S. H. VARGUS 7: Computed patterns from Markov processes. Behav. Sci, 1967b, 12, 323-328.

EVANS, S. H., \& ARNOULT, M. D. Schematic concept formation: Demonstration in a free sorting task. Psychon. Sci 1967, in press. ROSSER, E. M. Categorization and discrimination of tone sequences. Unpublished doctoral dissertation, Harvard, 1967.

Notes

1. This research was supported in part by TCU Research Foundation Grant No. PS 6672 . The research was previously reported at the Southwestern Psychological Association, April, 1967.

2. We have had inquiries regarding the availability of VARGUS 7 patterns. We have not as yet determined the most satisfactory method for supplying patterns in large quantities and would be happy to correspond with interested researchers about the method that would best suit their needs. Inquiries should be directed to Selby Evans. 